\title{
Retraction note: Invulnerability mechanism based on mobility prediction and opportunistic cloud computing with topological evolution for wireless multimedia sensor networks
}

\author{
Jianming Zhou ${ }^{1 *}$ and Tao Dong ${ }^{2}$
}

The authors are retracting this article [1]. The main contribution of this article is the prediction of node mobility. The proposed algorithm was derived based on the assumption of a linear channel response; however, the algorithm did not consider the wireless channel, which is usually non-linear with distance and forms random processes due to noise and interference. Additionally, the opportunistic cloud computational scheme proposed did not consider the motion and the velocity of the node.

\footnotetext{
Author details

${ }^{1}$ School of Information and Electronics, Beijing Institute of Technology, Beijing 100081, China. ${ }^{2}$ State Key Laboratory of Space-Ground Integrated Information Technology, Space Star Technology CO., Ltd, Beijing 100018, China.
}

Received: 15 December 2016 Accepted: 15 December 2016

Published online: 02 February 2017

\section{Reference}

1. Zhou, J, Dong, T. Invulnerability mechanism based on mobility prediction and opportunistic cloud computing with topological evolution for wireless multimedia sensor networks. EURASIP J. Wireless Commun. Netw. 2015243 (2015).

\footnotetext{
* Correspondence: zhoujm@bit.edu.cn

The online version of the original article can be found under doi:10.1186/ s13638-015-0471-6.

${ }^{1}$ School of Information and Electronics, Beijing Institute of Technology, Beijing 100081, China
} 